\title{
Ab interno gel implant in patients with primary open angle glaucoma and pseudoexfoliation glaucoma
}

\author{
Fritz H. Hengerer ${ }^{1,2,3^{*}}$, Gerd U. Auffarth ${ }^{1,2}$, Timur M. Yildirim ${ }^{1,2}$ and Ina Conrad-Hengerer ${ }^{1,2}$
}

\begin{abstract}
Purpose: To compare efficacy and safety results of an ab interno gel implant in patients with pseudoexfoliation glaucoma (PXG) and primary open angle glaucoma (POAG).

Methods: Retrospective analysis of the medical records of 110 consecutive eyes with open angle glaucoma who had received a XEN45 gel implant between March 2014 and June 2015. Intraocular pressure course, number of glaucoma medications, the need for additional intervention (including needling) and complications were evaluated until 12 months postoperatively.

Results: Data of 67 eyes with POAG and 43 eyes with PXG were analyzed. At 12 months postoperatively, the mean IOP had significantly decreased by $54.0 \%$ from preoperatively $31.85 \pm 8.5 \mathrm{mmHg}$ to $13.99 \pm 2.6 \mathrm{mmHg}$ in the POAG group, $(p=0.000$; Wilcoxon test), and by $55.2 \%$ from $31.63 \pm 9.0 \mathrm{mmHg}$ to $13.28 \pm 3.1 \mathrm{mmHg}$ in the PXG group ( $p=0.000$; Wilcoxon test). The mean number of anti-glaucoma medications had significantly decreased from 3. $25 \pm 0.8$ at baseline to $0.3 \pm 0.7$ medications at 12 months postoperatively in POAG eyes ( $p=0.000$; Wilcoxon test), and from $3.05 \pm 1.0$ to $0.3 \pm 0.6$ medications in PXG eyes ( $p=0.000$; Wilcoxon test). Hypotony (IOP $\leq 6$ $\mathrm{mmHg}$ ) was observed in 2 POAG eyes (3.0\%) and in 5 PXG eyes (11.7\%) at 1 month but normalized in all eyes at 12 months postoperatively. Severe complications were not observed. No statistically significant differences were found between PXG eyes and POAG eyes.

Conclusion: Our data indicate that the XEN45 gel implant provides significant and comparable reduction in IOP and anti-glaucoma medication during the one-year follow-up period in POAG as well as PXG eyes. This suggests that it may be a noteworthy alternative to traditional filtering procedures in patients with POAG and PXG respectively.
\end{abstract}

Keywords: Pseudoexfoliation glaucoma, Primary open angle glaucoma, Microinvasive glaucoma surgery, XEN45 gel implant

\section{Introduction}

Glaucoma, a progressive optic neuropathy leading to retinal ganglion cell loss and visual field defects, is still one of the leading causes of irreversible blindness [1, 2]. An elevated intraocular pressure (IOP) is a major risk factor for glaucoma development and progression and lowering IOP is still the only treatment option for glaucoma [3]. Surgical options may be taken into

\footnotetext{
* Correspondence: f.hengerer@buergerhospital-ffm.de

'Department of Ophthalmology, Ruprecht-Karls-University Heidelberg, 69120 Heidelberg, Germany

${ }^{2}$ International Vision Correction Research Center (IVCRC), University of

Heidelberg, 69120 Heidelberg, Germany

Full list of author information is available at the end of the article
}

account in patients whose IOP is not sufficiently controllable with two medications and have to be considered if maximally tolerated medical therapy fails [4]. Trabeculectomy is the gold standard filtering surgery with good efficacy; however, it requires a strict postoperative follow-up and has several intra- and postoperative complications [5-7]. In recent years, various micro-invasive glaucoma surgery (MIGS) techniques have been created to provide IOP reduction in a less invasive and safer way [8]. Of these, the XEN45 gel implant (XEN45) (Allergan, California USA) is the only commercially available MIGS device that uses the same outflow pathway as the surgical gold standard

(c) The Author(s). 2018 Open Access This article is distributed under the terms of the Creative Commons Attribution 4.0 International License (http://creativecommons.org/licenses/by/4.0/), which permits unrestricted use, distribution, and 
trabeculectomy by creating a permanent shunt from the anterior chamber to the subconjunctival space without the need of opening the conjunctiva [8-10]. It is a $6 \mathrm{~mm}$ long hydrophilic gelatin tube with an internal diameter of $45 \mu \mathrm{m}$ that had been designed to maximize long-term outflow while at the same time providing sufficient flow resistance to prevent hypotony [10-13]. Clinical studies have shown that the XEN45 gel implant provides a significant and sustained reduction in IOP and glaucoma medication with a low rate of complications [14-19]. However, most data were collected in patients with primary open angle glaucoma (POAG). In this context, it should be noted that in Europe, the indication of the XEN45 gel implant is limited for patients with POAG, whereas in the USA, the indication also includes the management of refractory glaucoma, including cases where previous surgical treatment has failed, cases of primary open angle glaucoma, and pseudoexfoliative or pigmentary glaucoma with open angles that are unresponsive to maximum tolerated medical therapy [20,21].

To our knowledge, this is the first study to evaluate and compare the efficacy of the XEN45 gel implant in POAG and pseudoexfoliation glaucoma (PXG) patients with inadequately controlled IOP despite maximized medical therapy or prior glaucoma surgery.

\section{Methods}

This is a retrospective analysis of consecutive patients with PXG and POAG who received a XEN45 gel implant between March 2014 and June 2015. The analysis included only the medical records of patients for whom data on all follow-up visits up to 12 months after surgery were available.

\section{Patients and assessments}

The medical records of 67 eyes with POAG and 43 eyes with PXG were included in the analysis. Patients with an inadequately controlled IOP and optic disc damage despite prior surgical intervention or maximum medication as well as an area of healthy, free and mobile conjunctiva in the target quadrant had received a XEN45 implant. Exclusion criteria were the same as for trabeculectomy: pregnancy, age $<18$ years, condition after pars plana vitrectomy, flat anterior chamber and narrow chamber angle. Preoperatively, a complete ophthalmic examination including gonioscopy had been performed. Postoperative evaluations were conducted at day 1 , week 1 , and months $1,3,6$, and 12 . At each visit, slit-lamp examination, gonioscopy, IOP assessment by Goldmann applanation tonometry (at each assessment, three measurements were performed, the mean value was recorded), as well as evaluation of the posterior pole were carried out. Moreover, the number of medications and adverse events were documented.

\section{Surgical technique}

All implantations were performed by one single surgeon (FHH) following a standardized implantation technique which has been described in detail in a recent publication [14]. The vast majority of XEN implants were performed as a stand-alone procedure. In brief, the XEN45 was implanted under peribulbar anesthesia using an ab interno approach. At first, a volume of $0.1 \mathrm{ml}$ of MMC solution $(0.01 \%$ mitomycin $\mathrm{C}$, a total dose of $10 \mu \mathrm{g})$ was injected subconjunctivally in the nasal superior quadrant to prevent further scarring of the conjunctiva. After the anterior chamber was filled with a medium grade viscoelastic device, the preloaded injector needle was then inserted through a $1.2 \mathrm{~mm}$ corneal paracentesis incision opposite the site of desired implantation. The needle was then directed across the anterior chamber and the injector tip was used to penetrate through the chamber angle above trabecular meshwork and the sclera at least $3 \mathrm{~mm}$ in length in order to place the implant properly. After careful removal of viscoelastic and hydration of paracenteses the eye was covered with a patch.

Postoperatively, topical antibiotics were given 4 times daily for 10 days in combination with steroids 6 times daily and tapered out over 6 weeks. Anti-glaucoma medication was given until surgery and was completely stopped after the implantation of the gel implant; there was no wash-out phase. At every visit, IOP was assessed and if IOP was elevated, additional anti-glaucoma medication or secondary intervention were given at the discretion of the surgeon. In case of conjunctival scarring and bleb failure due to Tenon's cyst formation, a needling procedure was performed under microscopic view in the operating room. The needling technique has recently been described in detail [14]. The administration of additional drugs during the needling was considered on a case-by-case basis. Only in cases of pronounced fibrosis (approx. 10\% of cases) $10 \mu \mathrm{g}$ mitomycin $\mathrm{C}$ was injected during needling, while in eyes with cystic fibroses no additional drugs were used.

\section{Statistical analysis}

Efficacy outcomes included IOP and the number of anti-glaucoma medications, and their changes as compared to baseline. Additional efficacy outcomes were target IOP of $\leq 18 \mathrm{mmHg}, \leq 15 \mathrm{mmHg}$ and $\leq 13 \mathrm{mmHg}$ at 12 months. Safety outcomes included hypotony rate (IOP $\leq 6$ $\mathrm{mmHg}$ ), rate of needling, as well as complications. Data were presented as mean and standard deviation, unless otherwise indicated. Baseline IOP was the IOP measured at the preoperative visit on medications. The IOP measured at each visit was then used to calculate the change from baseline. A Kaplan-Meier analysis was performed starting at 1 month postoperatively and using the following criteria for qualified success and complete success: 
Complete success was defined as an IOP reduction of at least $20 \%$ and an IOP value below $18 \mathrm{mmHg}$ without medication. Qualified success was defined as an IOP reduction of at least $20 \%$ and an IOP value below 18 $\mathrm{mmHg}$ with or without medication. In order to calculate differences between pre- and postoperative values, the parametric T-test was used. Also, the non-parametric Wilcoxon sign rank test was used for determining statistical significance within a group $(p<0.05$ considered statistically significant). For determining statistical significance between both groups, the non-parametric Mann-Whitney $U$ test and the $\mathrm{t}$-Test for independent samples were performed $(p<0.05$ considered statistically significant). In all other cases chi-square tests were applied $(p<0.05$ considered statistically significant).

\section{Results}

Overall, medical records of 110 eyes were included in this analysis. Of these, 67 eyes with POAG and 43 eyes with PXG had received a XEN45 gel implant. In the POAG group, the XEN stent was implanted in combination with cataract surgery in eight eyes, while 58 eyes received the XEN stent as standalone procedure. For one patient of the POAG group the information whether the XEN stent was implanted standalone or in combination with cataract surgery was missing. In the PEX group, nine eyes were treated with combined surgery, while 34 eyes received the XEN stent as standalone procedure. The number of eyes was stable at all postoperative visits in both groups. Demographic and baseline characteristics are displayed in Table 1. There was no statistically significant difference regarding demographic and baseline characteristics between the two groups $(p>0.05)$.

Both groups showed a significant reduction in IOP, which started on the first day after surgery and continued for the entire follow-up period of one year. At 12 months postoperatively, the mean IOP had significantly decreased by $54.0 \%$ from preoperatively $31.85 \pm 8.5$ $\mathrm{mmHg}$ to $13.99 \pm 2.6 \mathrm{mmHg}$ in the POAG group, $(p=$ 0.000 ; Wilcoxon test), and by $55.2 \%$ from $31.63 \pm 9.0$ $\mathrm{mmHg}$ to $13.28 \pm 3.1 \mathrm{mmHg}$ in the PXG group respectively ( $p=0.000$; Wilcoxon test). No significant differences between the two groups were detected at any time during follow-up observation $(p>0.085$ at each postoperative visit; $\mathrm{t}$-Test) (Fig. 1 ). The proportion of eyes per group that achieved target pressure values of $18 \mathrm{mmHg}, 15 \mathrm{mmHg}$ and $13 \mathrm{mmHg}$ one year postoperatively is shown in Fig. 2.

The anti-glaucoma medication was reduced in both groups in the median from preoperative 3 medications to 0 medications in the entire postoperative course. The mean number of anti-glaucoma medications had
Table 1 Demographics and baseline characteristics

\begin{tabular}{|c|c|c|}
\hline & $\begin{array}{l}\text { POAG } \\
(n=67)\end{array}$ & $\begin{array}{l}\text { PEXG } \\
(n=43)\end{array}$ \\
\hline $\begin{array}{l}\text { Age (years), } \\
\text { mean } \pm S D \text { (range) }\end{array}$ & $\begin{array}{l}69.6 \pm 13.7 \\
(34-91)\end{array}$ & $\begin{array}{l}74.0 \pm 8.3 \\
(51-89)\end{array}$ \\
\hline \multicolumn{3}{|l|}{ Gender, n (\%) } \\
\hline Male & $26(38.8)$ & $20(46.5)$ \\
\hline Female & $41(61.2)$ & $23(53.5)$ \\
\hline \multicolumn{3}{|l|}{ Operated Eye, n (\%) } \\
\hline OD (right eye) & $39(58.2)$ & $22(51.2)$ \\
\hline OS (left eye) & $28(41.8)$ & $21(48.8)$ \\
\hline $\begin{array}{l}\text { Cup to Disc Ratio, } \\
\text { mean } \pm \text { SD (range) }\end{array}$ & $\begin{array}{l}0.82 \pm 0.13 \\
(0.4-1.0)\end{array}$ & $\begin{array}{l}0.80 \pm 0.10 \\
(0.6-1.0)\end{array}$ \\
\hline \multicolumn{3}{|l|}{ Lens status, n (\%) } \\
\hline Phakic & 62 (92.5\%) & $39(90.7 \%)$ \\
\hline Pseudophakic & $5(7.5 \%)$ & $4(9.3 \%)$ \\
\hline \multicolumn{3}{|l|}{$\begin{array}{l}\text { Prior glaucoma } \\
\text { intervention, } \mathrm{n}(\%)\end{array}$} \\
\hline none & 22 (32.8\%) & $14(32.6 \%)$ \\
\hline Trabeculectomy & $13(19.4 \%)$ & $6(14.0 \%)$ \\
\hline Laser & $4(6.0 \%)$ & $0(0.0 \%)$ \\
\hline $\mathrm{CPC}$ & $13(19.4 \%)$ & $6(14.0 \%)$ \\
\hline Microstent ${ }^{a}$ & $10(14.9 \%)$ & $13(30.2 \%)$ \\
\hline Phacoemulsification & $5(7.5 \%)$ & $4(9.3 \%)$ \\
\hline $\begin{array}{l}\mathrm{IOP}(\mathrm{mmHg}) \\
\text { mean } \pm \mathrm{SD} \text { (range) }\end{array}$ & $\begin{array}{l}31.85 \pm 8.5 \\
(20-65)\end{array}$ & $\begin{array}{l}31.63 \pm 9.0 \\
(20-59)\end{array}$ \\
\hline $\begin{array}{l}\text { Number of medication, } \\
\text { mean } \pm S D \text { (range) }\end{array}$ & $\begin{array}{l}3.25 \pm 0.8 \\
(2-5)\end{array}$ & $\begin{array}{l}3.05 \pm 1.0 \\
(0-5)\end{array}$ \\
\hline
\end{tabular}

$P O A G$ primary open angle glaucoma, $P E X G$ pseudoexfoliation glaucoma, $C P C$ Cryophotocoagulation, IOP intraocular pressure, $S D$ standard deviation aother than XEN gel implant

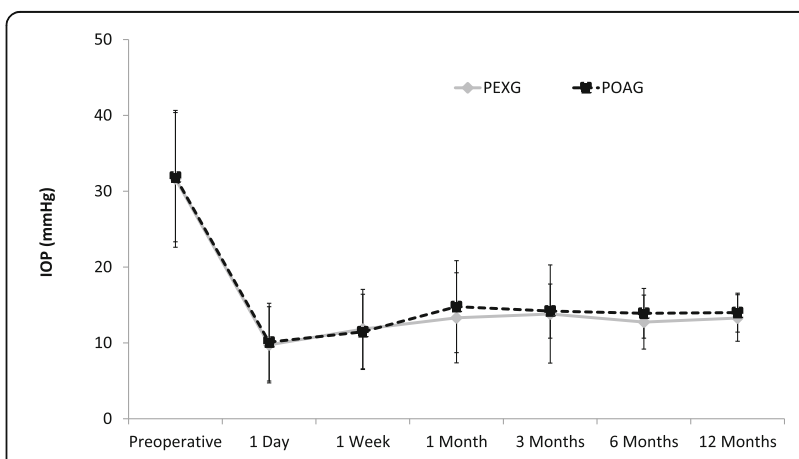

Fig. 1 Mean IOP at each study visit in PXG eyes $(n=43)$ und POAG eyes $(n=67)$ respectively. Error bars indicate SD for the mean. Within each group, the mean IOP was significantly reduced from baseline at any visit during the follow-up period ( $p=0.000$; Wilcoxon test). Between groups, no significant differences were observed $(p>0.085$ at each visit; t-Test) 


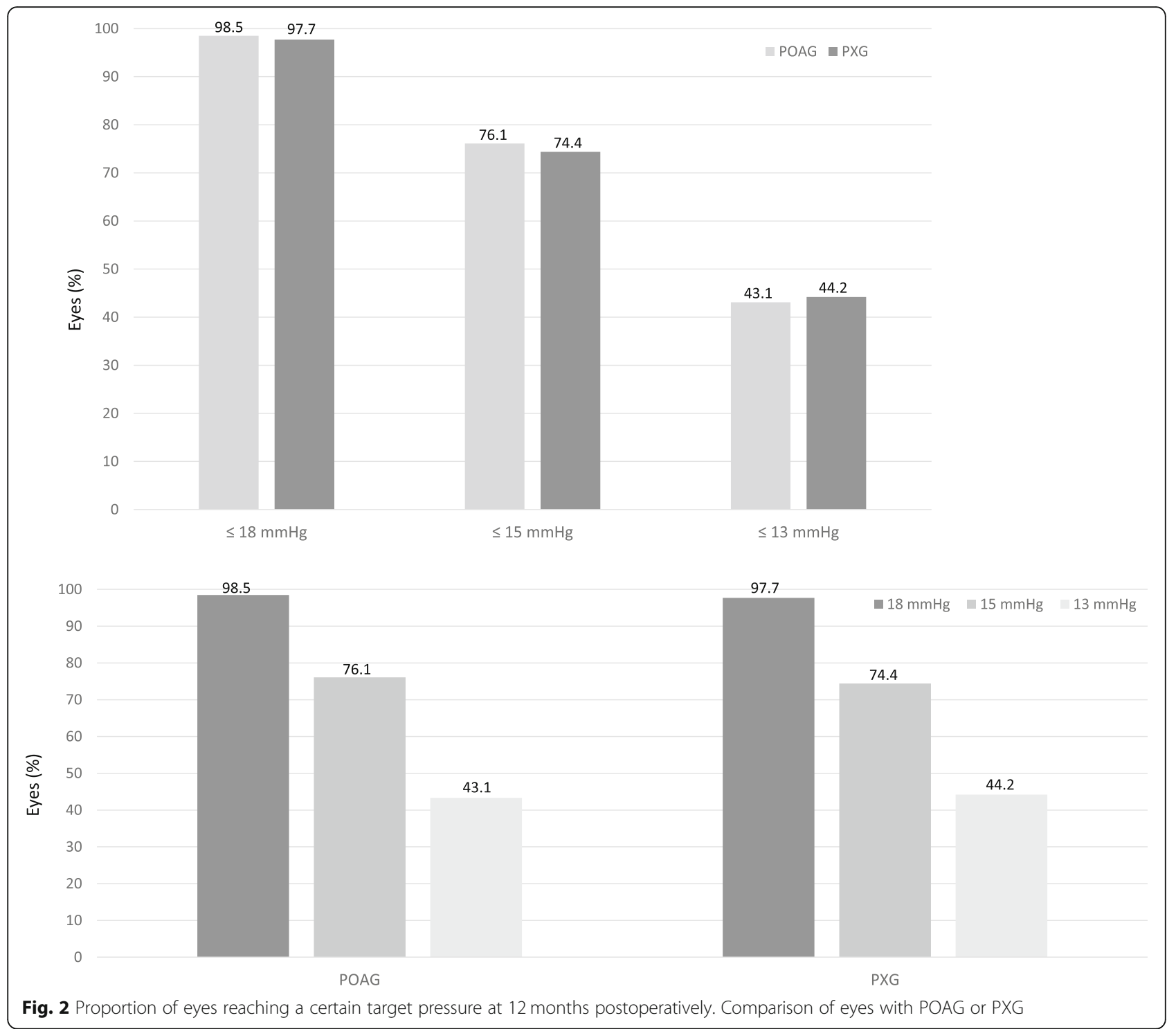

significantly decreased from $3.25 \pm 0.8$ at baseline to $0.3 \pm 0.7$ medications at 12 months postoperatively in POAG eyes ( $p=0.000$; Wilcoxon test), and from $3.05 \pm$ 1.0 to $0.3 \pm 0.6$ medications in PXG eyes $(p=0.000$; Wilcoxon test). No significant differences between the groups were observed at any time during follow-up ( $p>0,4$ at each postoperative visit) (Fig. 3). At 12 months postoperatively, $88.1 \%$ of POAG eyes and 83.1\% of PXG eyes were completely off drops.

One year after XEN45 implantation, $76.1 \%$ of eyes with POAG and $72.1 \%$ of those with PXG had achieved qualified success (IOP reduction of at least $20 \%$ and an IOP value below $18 \mathrm{mmHg}$ with or without medication). Complete success (IOP reduction of at least 20\% and an IOP value below $18 \mathrm{mmHg}$ without medication) was experienced by $64.2 \%$ of POAG eyes and by $55.8 \%$ of PXG eyes. The results of the Kaplan-Meier analysis using qualified success criteria and complete success criteria are shown in Figs. 4 and 5.

Overall, we had 28 cases of failure (i.e. IOP $>18 \mathrm{mmHg}$ and/or IOP reduction $<20 \%$; with or without medication) after 12 months due to reduced filtration by late fibrosis of the bleb area. Of these, there were 16 failures in the POAG group and 12 failures in the PXG group.

In the early postoperative phase, between week 1 and 3 months, $29.9 \%$ eyes in the POAG group required needling to enhance the outflow, while this was the case in $34.9 \%$ of PXG eyes respectively. The difference between both groups was not statistically significant $(p=0.058$; $\mathrm{t}$-Test). Twelve of 110 eyes received a single MMC injection between the first and third month (7 POAG eyes and 5 PXG eyes). Hypotony (IOP $\leq 6 \mathrm{mmHg}$ ) was observed in 2 POAG eyes (3.0\%) and 5 PXG eyes $(11.7 \%)$ at 1 month ( $p=0.108$; Fisher's exact test). 


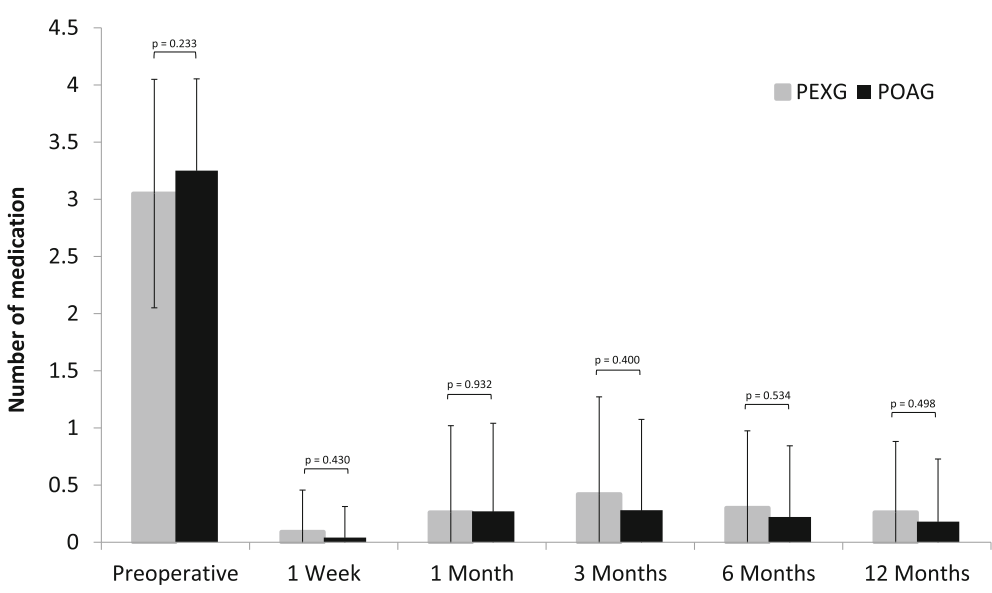

Fig. 3 Mean number of medication at each study visit in PXG eyes $(n=43)$ und POAG eyes $(n=67)$ respectively. Error bars indicate SD for the mean. Within each group, the mean number of medication was significantly reduced from baseline at any visit during the follow-up period ( $p=0.000$; Wilcoxon test). Between groups, no significant differences were observed ( $p>0,233$ at each visit)

Neither of the two groups had any cases of wound leakage, corneal alterations, device exposure or migration, choroidal effusion or hemorrhage, choroidal detachment, or endophthalmitis during the follow-up.

\section{Discussion}

The purpose of our retrospective data analysis was to evaluate and compare the efficacy and safety results of the XEN45 gel implant in patients with PXG and
POAG. Within the last years, various MIGS devices have been developed with the aim to provide less invasive methods of decreasing IOP in glaucoma patients than traditional surgery and reducing the patients' dependency on topical medications [8]. Several clinical studies have shown that the XEN45 gel implant provides a significant and enduring reduction of IOP and anti-glaucoma medication [14-19]. Since these studies have focused on POAG patients, very little data is

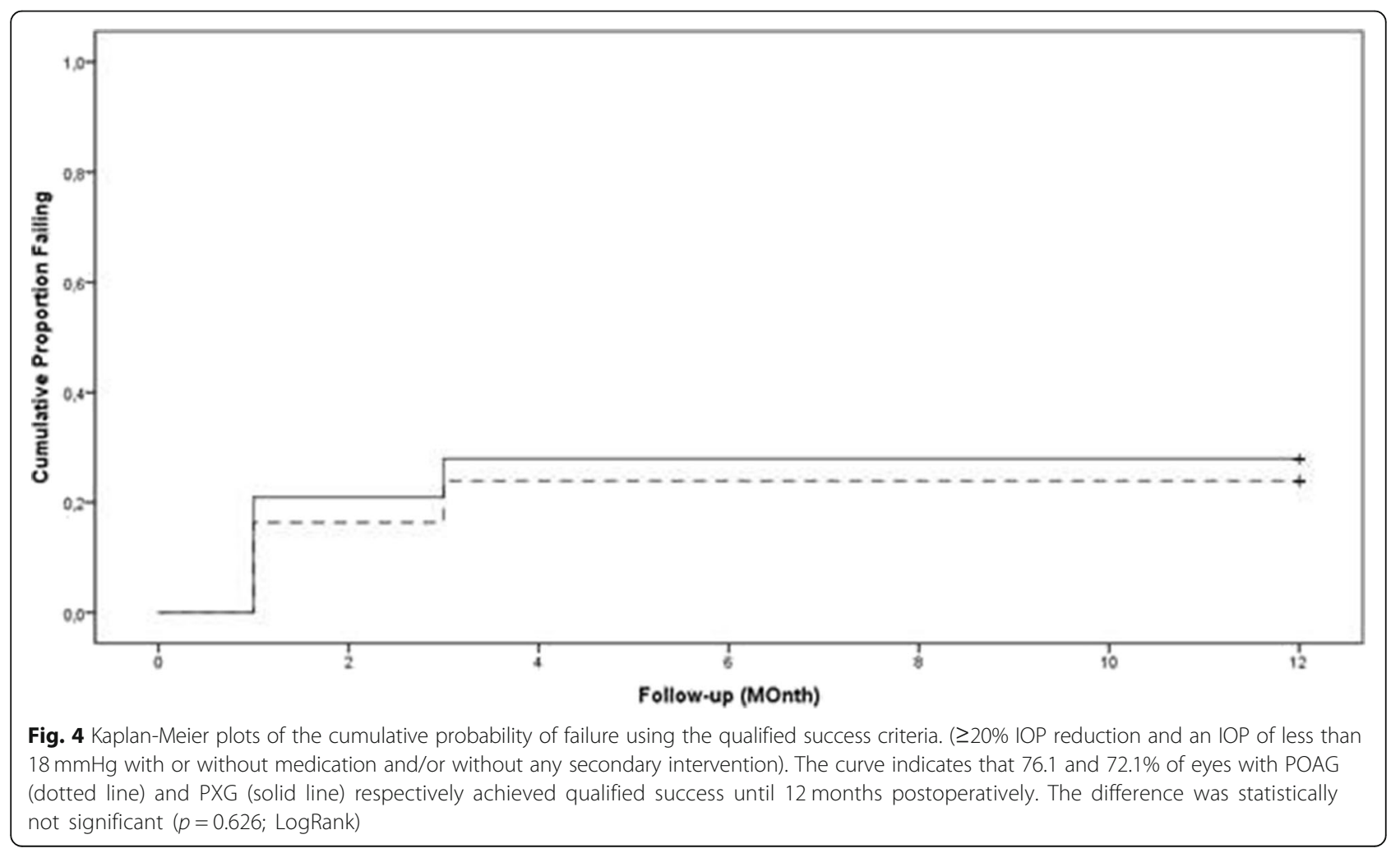




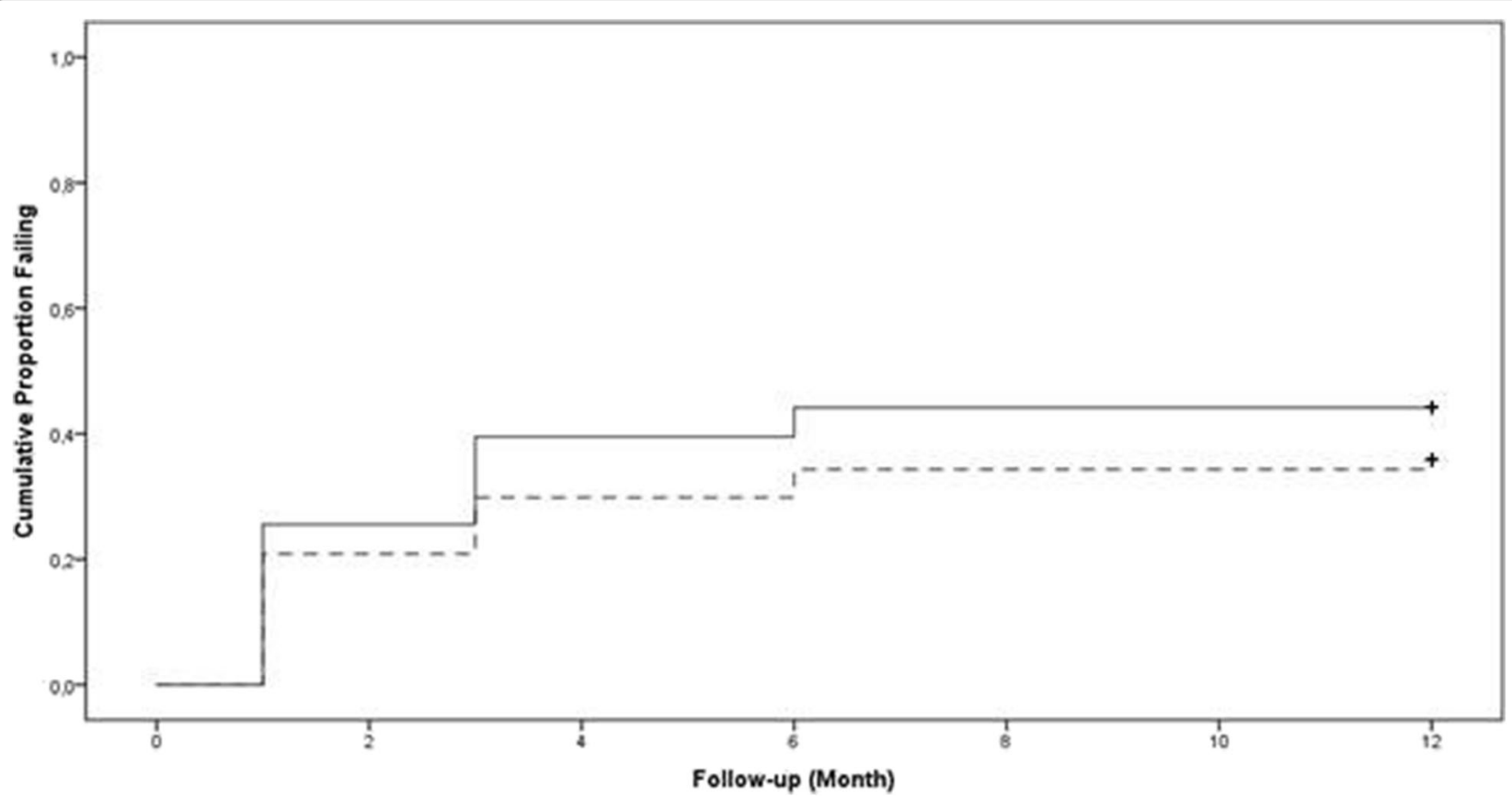

Fig. 5 Kaplan-Meier plots of the cumulative probability of failure using the complete success criteria. ( $\geq 20 \%$ IOP reduction and an IOP of less than $18 \mathrm{mmHg}$ with or without medication and/or without any secondary intervention). The curve indicates that 64.2 and $55.8 \%$ of eyes with POAG (dotted line) and PXG (solid line) respectively achieved complete success until 12 months postoperatively. The difference was statistically not significant $(p=0.374$; LogRank)

available on PXG, the most common secondary glaucoma [22]. However, because of the aggressive course of the disease and their poor response to medical therapy [23], PXG patients in particular could probably benefit from an early and less invasive intervention.

Our results show that in both patient groups a strong and comparable IOP decrease as well as reduction of anti-glaucoma medication can be achieved with the XEN45 gel implant. In POAG eyes as well as in PXG eyes, we observed a significant reduction in the mean IOP by more than $50 \%$ to a mean IOP below $15 \mathrm{mmHg}$ at each postoperative visit. In both groups, the IOP lowering effect started on the first postoperative day and continued throughout the follow-up period. Additionally, in both patient groups the mean number of anti-glaucoma medications was significantly reduced after XEN45 implantation and more than $80 \%$ of eyes were completely off drops. This substantial reduction of anti-glaucoma medication achieved in both patient groups may contribute to a better quality of life and can increase patients' satisfaction with their therapy [24]. At the same time, the XEN45 had a reliable safety profile with no severe complications. We did not observe any significant differences regarding efficacy and safety between the two groups. However, even if the differences were not statistically significant, hypotony of less than $6 \mathrm{mmHg}$ was more frequent in PXG eyes one month postoperatively. This may be due to the fact that some pseudoexfoliative material has been removed by rinsing the anterior chamber during implantation of the XEN45, thus improving fluid drainage. Nevertheless, all cases of hypotony were resolved after six months and no choroidal effusion was observed.

A total of 17 eyes in our study received the XEN implant in combination with cataract surgery (8 POAG eyes, 9 PXG eyes). With regard to IOP and number of medications at baseline and after 12 months we could not find any statistical differences between the different subgroups, however, since the number of combined cases is very low ( $<10$ in the respective subgroups), these results should be viewed with the utmost caution and should not be overinterpreted.

Our results are well in line with data from other clinical studies evaluating the efficacy and safety of the XEN45 gel implant in patients with POAG [14-19]. Moreover, our results, collected in 43 eyes with PXG, a very similar to the data from Ilveskoski and Tuuminen who report clinical results of XEN45 gel implant in 10 patients with PXG. In this group of patients, the mean IOP was reduced by $47.4 \%$ from $33 \mathrm{mmHg}$ preoperatively to $10.2 \mathrm{mmHg}$ at six months postoperatively and mean medication had decreased from 2.4 to 0.9 medications [25].

As the XEN45 gel implant uses the same outflow path as the surgical gold standard trabeculectomy, our study results are not comparable to those of other MIGS 
devices which target the natural outflow pathways of aqueous humor. Overall, our results indicate a strong IOP-lowering effect after XEN45 implantation which can be achieved in POAG patients as well as in PXG patients and is almost comparable to that of conventional filtrating glaucoma surgery [6], although however, in the absence of a prospective, randomized trial, it is difficult to draw any major conclusions versus trabeculectomy. At the same time, typical safety risks of traditional incisional glaucoma surgery, such as hypotony-related complications, scarring, foreign body reaction, cataract formation, and surgically induced astigmatism are almost negligible in this ab-interno procedure. Therefore, given the marked and enduring IOP-lowering effect of the XEN45 and its reliable safety profile, this micro-invasive procedure might be considered at an earlier stage of glaucoma disease than conventional filtrating glaucoma surgery. Especially in PXG patients, who often respond only weakly to medical therapy and exhibit rapid disease progression [23], this approach may help to achieve a low target IOP with reduced medication at an earlier stage. Moreover, this strategy would preserve the conjunctiva if further glaucoma filtrating surgery interventions might be required to control IOP more efficiently.

Our study has limitations that should be addressed. First, it was a retrospective analysis of single center data, however, this setting reflects the everyday clinical routine and thus provides important insights into the efficiency and safety of the XEN45 gel implant in everyday clinical practice. Secondly our results are only out to 12 months which is a relatively short time in a lifetime chronic disease. Thirdly no consistent follow up of perimetry data was available, as preoperative perimetry assessments had been performed in the respective private practices, before the patients were referred to our clinic. Nevertheless, all patients appeared at our department with clinical signs of progression or uncontrolled IOP values, respectively.

However, despite its limitations, this study contributes to increasing evidence showing the safety and efficacy of the XEN45 gel implant. This procedure provided significant and comparable reduction in IOP and anti-glaucoma medication during the entire follow-up period in POAG and PXG eyes, suggesting that it might be a noteworthy alternative to traditional filtering procedures in patients with POAG and PXG respectively. To confirm these one-year results, further prospective, randomized studies with longer follow-ups are required to evaluate the efficacy and safety of this procedure in PXG patients.

\section{Abbreviations}

CPC: Cryophotocoagulation; IOP: Intraocular pressure; MIGS: Minimally/ Micro-invasive glaucoma surgery; MMC: Mitomycin C; POAG: Primary open angle glaucoma; PXG: Pseudoexfoliation glaucoma
Acknowledgements

This publication was supported by an independent publication grant from Allergan.

Funding

This publication was supported by an independent publication grant from Allergan.

Availability of data and materials

The datasets used and/or analyzed during the current study are available from the corresponding author on reasonable request.

\section{Authors' contributions}

FHH was involved in data collection. FHH and ICH were involved in data analysis as well as drafting the manuscript. GUA and TY made substantial contributions to conception of the manuscript and were involved in data interpretation and revising the manuscript critically for important intellectual content. All authors read and approved the final manuscript.

Ethics approval and consent to participate

The data documentation and retrospective analysis was approved by the local Ethics Committee and adhered to the tenets of the Declaration of Helsinki. According to the approval of the Ethics Committee, the data for this retrospective analysis were evaluated pseudonymized and no patient informed consent was required.

Consent for publication

Not applicable.

\section{Competing interests}

The authors declare that they have no competing interests.

\section{Publisher's Note}

Springer Nature remains neutral with regard to jurisdictional claims in published maps and institutional affiliations.

\section{Author details}

'Department of Ophthalmology, Ruprecht-Karls-University Heidelberg, 69120 Heidelberg, Germany. ${ }^{2}$ International Vision Correction Research Center (IVCRC), University of Heidelberg, 69120 Heidelberg, Germany. ${ }^{3}$ University Eye Hospital, Ruprecht-Karls-University, Im Neuenheimer Feld 400, 69120

Heidelberg, Germany.

Received: 8 March 2018 Accepted: 3 December 2018

Published online: 27 December 2018

\section{References}

1. Cvenkel B, Kontestabile AS. Correlation between nerve fibre layer thickness measured with spectral domain OCT and visual field in patients with different stages of glaucoma. Graefes Arch Clin Exp Ophthalmol. 2011;249: $575-84$

2. Tham YC, Li X, Wong TY, Quigley HA, et al. Global prevalence of glaucoma and projections of glaucoma burden through 2040: a systematic review and meta-analysis. Ophthalmology. 2014;121:2081-90.

3. Heijl A, Leske MC, Bengtsson B, Early Manifest Glaucoma Trial Group, et al. Reduction of intraocular pressure and glaucoma progression: results from the Early Manifest Glaucoma Trial. Arch Ophthalmol. 2002;120:1268-79.

4. European Glaucoma Society (EGS). Terminology and guidelines for glaucoma. 4th ed; 2014

5. Gedde SJ, Herndon LW, Brandt JD, et al. Tube versus trabeculectomy study group. Postoperative complications in the tube versus trabeculectomy (TVT) study during five years of follow-up. Am J Ophthalmol. 2012;153:804-14.

6. Gedde SJ, Schiffman JC, Feuer WJ, et al. Tube versus trabeculectomy study group. Treatment outcomes in the tube versus trabeculectomy (TVT) study after five years of follow-up. Am J Ophthalmol. 2012;153:789-803.

7. Jampel HD, Musch DC, Gillespie BW, et al. Perioperative complications of trabeculectomy in the collaborative initial glaucoma treatment study (CIGTS). Am J Ophthalmol. 2005;140:16-22.

8. Richter GM, Coleman AL. Minimally invasive glaucoma surgery: current status and future prospects. Clin Ophthalmol. 2016:10:189-206.

9. Vera VI, Horvath C. XEN gel stent: the solution designed by Aquesys. In: Samples JR, Ahmed IIK, editors. Surgical innovations in glaucoma. New York: Springer Science + Business Media NY; 2014. p. 189-98. 
10. Lewis RA. Ab interno approach to the subconjunctival space using a collagen glaucoma stent. J Cat Refract Surg. 2014;40:1301-6.

11. Sheybani A, Reitsamer H, Ahmed IIK. Fluid dynamics of a novel micro-fistula implant for the surgical treatment of glaucoma. Invest Ophthalmol Vis Sci. 2015;56:4789-95.

12. Yu D-Y, Morgan WH, Sun $X$, et al. The critical role of the conjunctiva in glaucoma filtration surgery. Prog Retin Eye Res. 2009;28:303-28.

13. Sheybani A, Dick B, Ahmed III. Early clinical results of a novel ab interno gel stent for the surgical treatment of open-angle glaucoma. J Glaucoma. 2016;25:e691-6.

14. Hengerer FH, Kohnen T, Mueller M, Conrad-Hengerer I. Ab interno gel implant for the treatment of glaucoma patients with or without prior glaucoma surgery - one year results. J Glaucoma. 2017;26:1130-6.

15. Grover DS, Flynn WJ, Bashford KP, Lewis RA, Duh YJ, Nangia RS, Niksch B. Performance and safety of a new ab interno gelatin stent in refractory glaucoma at 12 months. Am J Ophthalmol. 2017;183:25-36.

16. Galal A, Bilgic A, Eltanamly R, Osman A. XEN glaucoma implant with mitomycin C 1-year follow-up: result and complications. J Ophthalmol. 2017;2017:5457246.

17. De Gregorio A, Pedrotti E, Russo L, Morselli S. Minimally invasive combined glaucoma and cataract surgery: clinical results of the smallest ab interno gel stent. Int Ophthalmol. 2017. https://doi.org/10.1007/s10792-017-0571-x [Epub ahead of print].

18. Sheybani A, Ahmed IK. Ab interno gelatin stent with mitomycin-C combined with cataract surgery for treatment of open-angle glaucoma: 1 year results: Presented at ASCRS; 2015.

19. Barton K, Sng C, Vera V. XEN45 TM implantation for primary open-angle glaucoma: one-year results of a multicenter study: Poster presented at the ASCRS; 2016. p. 6.01

20. Directions for use for the XEN 45 GLAUCOMA TREATMENT SYSTEM. Europe.

21. Directions for use for the XEN 45 GLAUCOMA TREATMENT SYSTEM. U.S.

22. Aboobakar IF, Johnson WM, Stamer WD, Hauser MA, Allingham RR. Major review: exfoliation syndrome; advances in disease genetics, molecular biology, and epidemiology. Exp Eye Res. 2017;154:88-103.

23. Brooks AMV, Gillies WE. The presentation and prognosis of glaucoma in pseudoexfoliation of the lens capsule. Ophthalmology. 1988;95:271-6.

24. Quaranta L, Riva I, Gerardi C, et al. Quality of life in glaucoma: a review of the literature. Adv Ther. 2016;33:959-81.

25. Ilveskoski L, Tuuminen R. Combined microinvasive glaucoma and cataract surgery in patients with pseudoexfoliation glaucoma: clinical results of a gel stent. J Cataract Refract Surg. 2017;43:1124-5.

Ready to submit your research? Choose BMC and benefit from:

- fast, convenient online submission

- thorough peer review by experienced researchers in your field

- rapid publication on acceptance

- support for research data, including large and complex data types

- gold Open Access which fosters wider collaboration and increased citations

- maximum visibility for your research: over $100 \mathrm{M}$ website views per year

At $\mathrm{BMC}$, research is always in progress.

Learn more biomedcentral.com/submissions 